\title{
SOLVER como suplemento do Excel
}

\author{
SOLVER as Excel add-in
}

\section{SOLVER como suplemento de Excel}

A ferramenta SOLVER é extremamente útil para todos os profissionais que necessitam resolver problemas de recursos limitados e usá-los da maneira mais eficiente possivel; normalmente procurando Maximização de Lucros e Minimização de Custos.

A otimização matemática conduz a determinação de objetivos, considerando-se as restrições para todas as variáveis de decisão.

Com isso, a cessão do SOLVER pela Frontline Systems à Microsoft, faz deste aplicativo um excelente solucionador de problemas.
The tool solver is extremely useful for all professionals who need to solve problems of limited resources and use them in the most efficient manner possible; normally looking for maximization of profits and minimizing costs. The mathematical optimization leads the determination of goals, considering the restrictions for all variables of the decision. With this, the transfer of the SOLVER by Frontline systems to Microsoft, makes this application a excellent troubleshooter.
Palavras-chave: Desenvolvimentismo.

\section{Autores: \\ Marco Antonio Greco}

Engenheiro Mecânico (FEI-PUC/SP - USU/RJ). Pós-graduado em Engenharia Econômica e Administração Industrial (COPPE-UFRJ). Professor do Curso de Engenharia Mecânica na Universidade Estácio de Sá - Campus Praça XI e Campus Niterói

Email:marco.grecog@gmail.com

Desde muito tempo que os empresários, financistas, contabilistas, responsáveis por produção nas indústrias, logísticas de distribuição em todos os países, se preocupam com os Lucros, com os Custos dos processos de fabricação, de distribuição e das vendas.

Os profissionais que se incumbem de preparar dados para obter resultados das necessárias análises, normalmente, são Economistas, Contabilistas, Administradores, Engenheiros e muitas vezes Analistas de Sistemas.

Atualmente com a larga utilização de micros, cada vez mais potentes em processamento e com grande capacidade de armazenamento, ferramentas desenvolvidas por empresas fornecedoras de soluções de Informática, tornam disponíveis software que facilitam e agilizam os cálculos para se determinar objetivos a alcançar.

Uma dessas ferramentas como o nome de aplicativo, programa ou rotina está disponível em uma das plataformas de planilhas eletrônicas mais utilizadas no mundo, o Excel, do Office da Microsoft.

Trata-se do "SOLVER"; um suplemento instalável do Excel, é um software desenvolvido pela Frontline Systems e cedido à Microsoft para utilização naquela ferramenta do Office.

A Frontline Systems (www.frontsys.com) possui software para bancos de dados (matrizes) muito grandes, bem superiores aos possíveis com o Excel.

Para o bom aproveitamento desta ferramenta é desejável, mas não imprescindível, que o usuário tenha noções de Álgebra Linear, Matrizes e Programação Linear.

A montagem das matrizes, a análise das restrições e a determinação da função objetivo levam à solução desejada.

$\mathrm{Na}$ maioria dos cursos de Pós-graduação em Gestão de Negócios, Engenharia Econômica, Administração Industrial, Logística, etc. oferecidos pelas Universidades; este software é utilizado para resolver problemas de Maximização de Lucros, Minimização de Custos e outros.

O objetivo deste artigo é somente mostrar alguns exemplos de problemas que foram resolvidos com o uso do SOLVER; para que os leitores vejam com calma e aproveitem os exemplos para quando precisarem. 
Este $1^{\circ}$ exemplo tem a ver com o orçamento da publicidade alocada em função da sazonabilidade para se verificar o Lucro.

A empresa do Sr. Jorge Bobina apresenta um modelo de Marketing típico mostrando as vendas partindo de um número básico (talvez devido à equipe de vendas) juntamente com aumentos em publicidade, mas com retorno decrescente; por exemplo, os primeiros $\mathbf{R} \$ \mathbf{1 0 . 0 0 0 , 0 0}$ de publicidade no $1^{\circ}$ trimestre produzem cerca de 798 unidades vendidas a mais, mas os próximos $R \$ 10.000,00$ produzem apenas cerca de 399 unidades a mais. Verificar se o orçamento de publicidade está baixo demais e se a publicidade deveria ser alocada de modo diferente ao longo do tempo para se tirar proveito do fator sazonabilidade.

\begin{tabular}{|c|c|c|c|c|c|}
\hline \multicolumn{6}{|c|}{ Problema de Marketing com Solver } \\
\hline Mês & $\mathbf{1}^{\circ}$ Trim & $2^{\circ}$ Trim & $3^{\circ}$ Trim & $4^{\circ}$ Trim & Total \\
\hline Sazonalidade & 0,9 & 1,1 & 0,9 & 1,4 & \\
\hline Unidades vendidas & 3.592 & 4.390 & 3.592 & 5.587 & 17.160 \\
\hline Receita de vendas & $143.662,10$ & $\mathbf{1 7 5 . 5 8 7 , 0 2}$ & $143.662,10$ & $223.474,38$ & 686.386 \\
\hline Custo de vendas & $89.788,81$ & $109.741,88$ & $89.788,81$ & $139.671,49$ & 428.991 \\
\hline Margem bruta & $53.873,29$ & $65.845,13$ & $53.873,29$ & $83.802,89$ & 257.395 \\
\hline Equipe de vendas & $8.000,00$ & $8.000,00$ & $8.000,00$ & $8.000,00$ & 32.000 \\
\hline Publicidade & $10.000,00$ & $10.000,00$ & $10.000,00$ & $10.000,00$ & 40.000 \\
\hline Despesas administrativas & $21.549,32$ & $26.338,05$ & $21.549,32$ & $33.521,16$ & 102.958 \\
\hline Custo total & $39.549,32$ & $44.338,05$ & $39.549,32$ & $51.521,16$ & 174.958 \\
\hline Lucro do produto & $14.323,97$ & $21.507,08$ & $14.323,97$ & $32.281,74$ & $82.436,76$ \\
\hline Margem de lucro & $10 \%$ & $12 \%$ & $10 \%$ & $14 \%$ & $12 \%$ \\
\hline Preço do produto & 40,00 & & & & \\
\hline Custo do produto & 25,00 & & & & \\
\hline
\end{tabular}


$\mathrm{O} 2^{\circ}$ exemplo refere-se à programação de uma dieta do Sr. Rolando Escada Abaixo, levando em conta as quantidades de vitaminas e os alimentos que as contém, considerando os custos unitários dos alimentos com objetivo de saber o consumo diário dos alimentos e ao menor custo possível.

O Sr. Rolando Escada Abaixo foi forçado pelo seu médico, Dr. Fujiu Nakombi, a fazer uma dieta alimentar que forneça diariamente, pelos menos as seguintes quantidades de vitaminas A, B, C e D:

\begin{tabular}{|c|c|}
\hline A & $\mathbf{8 0}$ \\
\hline B & $\mathbf{7 0}$ \\
\hline C & $\mathbf{1 0 0}$ \\
\hline D & $\mathbf{6 0}$ \\
\hline
\end{tabular}

A dieta deverá incluir leite, arroz, feijão e carne, que contêm os seguintes miligramas de vitaminas em cada uma de suas unidades

\begin{tabular}{|c|c|c|c|c|}
\hline & \multicolumn{4}{|c|}{ Alimentos } \\
\hline Vitaminas & Leite & Arroz & Feijão & Carne \\
\hline A & $\mathbf{1 0}$ & $\mathbf{5}$ & $\mathbf{9}$ & $\mathbf{1 0}$ \\
\hline B & $\mathbf{8}$ & 7 & $\mathbf{6}$ & $\mathbf{6}$ \\
\hline C & $\mathbf{1 5}$ & $\mathbf{3}$ & $\mathbf{4}$ & $\mathbf{7}$ \\
\hline D & $\mathbf{2 0}$ & 2 & $\mathbf{3}$ & $\mathbf{9}$ \\
\hline
\end{tabular}

Os custos unitários desses alimentos são os seguintes :

\begin{tabular}{|l|l|}
\hline Leite & $\$ 1,00 / \mathbf{l}$ \\
\hline Arroz & $\$ 0,80 / \mathbf{~ k g}$ \\
\hline Feijão & $\$ 1,20 / \mathbf{~ k g}$ \\
\hline Carne & $\$ 3,50 /$ kg \\
\hline
\end{tabular}

Deseja-se saber o consumo diário de cada um desses alimentos de tal maneira que a dieta satisfaça as prescrições médicas e seja a de menor custo possível.

$\mathbf{x j}=$ quantidades de produtos que irão entrar na dieta

Objetivo : Minimizar : 1.x1 + 0,8.x2 + 1.2.x3 + 3,5.x4

Restrições :

$10 . \times 1+5 . \times 2+9 . x 3+10 . x 4>=80$

$8 . \times 1+7 . \times 2+6 . x 3+6 . x 4>=70$

$15 . \times 1+3 . x 2+4 . x 3+7 . x 4>=100$

$20 \times x 1+2 \cdot x 2+3 \cdot x 3+9 \cdot x 4>=60$

$\mathbf{x j}>=\mathbf{0}$

\begin{tabular}{|c|r|c|c|c|c|}
\hline \multicolumn{2}{|c|}{ Quantidades de vitaminas ==> } & 80 & 70 & 100 & 60 \\
\hline \hline A & 2650 & 10 & 5 & 9 & 10 \\
\hline B & 2090 & 8 & 7 & 6 & 6 \\
\hline C & 2230 & 15 & 3 & 4 & 7 \\
\hline D & 2580 & 20 & 2 & 3 & 9 \\
\hline & Custos unitários ==> & 1,00 & 0,80 & 1,20 & 3,50 \\
\hline & 466,00 & & & & \\
\hline
\end{tabular}


O $3^{\circ}$ problema é o interesse do empresário $\mathrm{Mc}^{\prime}$ Akon em maximizar o lucro mensal da produção de seus produtos, com os recursos que têm disponíveis.

A empresa do Sr. Mc' Akon está interessada em maximizar o lucro mensal proveniente de 4 de seus produtos, designados por P1, P2, P3 e P4. Para fabricar esses 4 produtos, a fábrica utiliza 2 tipos de máquinas, M1 e M2 e 2 tipos de mão de obra, MO1 e MO2.

Quadro de disponibilidades

\begin{tabular}{|c|c|c|c|c|c|}
\hline Máquina & \multicolumn{2}{|c|}{ Tempo disponível } & Mão de obra & \multicolumn{2}{|l|}{ Tempo disponível } \\
\hline \multicolumn{2}{|c|}{ (maq.hora/mês) } & & \multicolumn{2}{|l|}{ (homem.hora/mês) } \\
\hline M1 & $\mathbf{8 0}$ & & MO1 & 120 & \\
\hline M2 & 20 & & MO2 & 160 & \\
\hline
\end{tabular}

$\mathrm{N}^{0}$ de máq.-hora para produzir uma unidade de cada produto

\begin{tabular}{|c|c|c|c|c|}
\hline & \multicolumn{4}{|c|}{ Produtos } \\
\hline Máq. & P1 & P2 & P3 & P4 \\
\hline M1 & 5 & 4 & 8 & 9 \\
\hline M2 & 2 & 6 & 0 & 8 \\
\hline
\end{tabular}

$\mathrm{N}^{0}$ de homens-hora para produzir uma unidade de cada produto

\begin{tabular}{|c|c|c|c|c|}
\hline Mão de & \multicolumn{4}{|c|}{ Produtos } \\
\hline obra & P1 & $\mathbf{P 2}$ & P3 & P4 \\
\hline MO1 & 2 & 4 & 2 & 8 \\
\hline MO2 & 7 & 3 & 0 & 7 \\
\hline
\end{tabular}

Potencial de vendas Lucro unitário

\begin{tabular}{|c|c|c|c|}
\hline Produtos & \multicolumn{2}{|c|}{ (unidades/mês) } & (\$/unidade) \\
\hline P1 & $\mathbf{7 0}$ & & $\mathbf{1 0}$ \\
\hline P2 & $\mathbf{6 0}$ & & $\mathbf{8}$ \\
\hline P3 & $\mathbf{4 0}$ & & $\mathbf{9}$ \\
\hline P4 & $\mathbf{2 0}$ & & $\mathbf{7}$ \\
\hline
\end{tabular}

$\mathbf{x j}$ = produção mensal de cada produto $\mathbf{P j}$

Objetivo : maximizar 10.x1 + 8.x2 + 9.x3 + 7.x4

Restrições :

$5 . \times 1+4 . \times 2+8 . x 3+9 . \times 4<=80$

$2 . x 1+6 . x 2+\quad+8 . x 4<=20$

$2 . x 1+4 . x 2+2 . x 3+8 . x 4<=120$

$7 . \times 1+3 . \times 2+\quad+7 . \times 4<=160$

$x 1<=70 ; x 2<=60 ; x 3<=40 ; x 4<=20$

\begin{tabular}{|c|c|c|c|c|c|c|}
\hline & \multicolumn{5}{|c|}{ Produtos } & \multirow[b]{2}{*}{20} \\
\hline & Potenci & ndas & 70 & 60 & 40 & \\
\hline M1 & 80 & 217 & 5 & 4 & 8 & 9 \\
\hline M2 & 20 & 124 & 2 & 6 & 0 & 8 \\
\hline M01 & 120 & 126 & 2 & 4 & 2 & 8 \\
\hline MO2 & 160 & 143 & 7 & 3 & 0 & 7 \\
\hline $\begin{array}{c}\mathbf{x j}=\text { Produção } \\
\text { mensal } \\
\text { de cada } \\
\text { produto }\end{array}$ & & & 10 & 8 & 9 & 7 \\
\hline & & & $1.680,00$ & & & \\
\hline
\end{tabular}


O $4^{\circ}$ problema também é de um empresário, Sr. Omar Motta, que e armazena seus produtos (garrafas de suco e garrafas de vinho) para venda, considerando restrições para os 2 tipos de produtos, ele quer determinar o lucro líquido máximo com a produção da fábrica.

A fábrica do Sr. Omar Motta produz garrafas e usa uma única máquina, que está disponível 60 horas/semana

O trabalho é programado semanalmente onde se gasta toda a semana produzindo garrafas e armazenando-as em um galpão; ao final de cada semana toda a produção da semana é despachada Dois produtos são produzidos, garrafas de suco e garrafas de vinho, a máquina gasta 6 horas para produzir 100 caixas de de garrafas de suco e 5 horas para produzir 100 caixas de garrafas de vinho. $O$ galpão tem $15000 \mathrm{~m}^{3}$. A contribuição líquida por caixa de garrafas de suco para o lucro (sem custos de produção) é $\$ 5,00$ e é de $\$ 4,50$ por caixa de garrafas de vinho. $O$ departamento de Marketing estima que que se pode vender quantas caixas de garrafas de vinho se puder produzir, mas se pode vender um máximo de 800 caixas de garrafas de suco por semana. Deve ser determinada a produção da fábrica, que respeitando as restrições produzirá o lucro líquido máximo

Formulação algébrica :

$\mathbf{J}=\mathbf{n}^{0}$ de caixas de garrafas de suco produzidas por semana

$\mathbf{W}=\mathbf{n}^{\circ}$ de caixas de garrafas de vinho produzidas por semana

Maximizar o lucro total líquido em $\$: 5^{*} \mathrm{~J}+4,5^{*} \mathrm{~W}$

Restrições :

Tempo de máquina em horas : $(6 / 100) * J+(5 / 100) * W<=60$

Venda de garrafas de suco, em caixas : $\mathrm{J}<=800$

Não-negatividade : $\mathrm{J}, \mathrm{W}>=\mathbf{0}$

A seguir : problema montado com os dados enunciados

\begin{tabular}{|c|c|c|c|c|c|c|c|}
\hline $\begin{array}{l}\text { Tipo da } \\
\text { garrafa }\end{array}$ & $\begin{array}{c}\text { Horas de } \\
\text { trabalho para } \\
\text { cada caixa de } \\
\text { garrafas }\end{array}$ & $\begin{array}{c}\text { Espaço para } \\
\text { armazenar } \\
\text { cada caixa }\end{array}$ & $\begin{array}{c}\text { Contribuição } \\
\text { líquida por } \\
\text { caixa }\end{array}$ & $\begin{array}{l}\text { Número } \\
\text { de caixas }\end{array}$ & $\begin{array}{l}\text { Lucro total } \\
\text { de garrafas }\end{array}$ & $\begin{array}{c}\text { Espaço } \\
\text { necessário } \\
\text { total }\end{array}$ & $\begin{array}{c}\text { Total } \\
\text { horas } \\
\text { trabalhadas }\end{array}$ \\
\hline Suco $=\mathbf{J}$ & 0,06 & 10 & $\mathbf{5 , 0}$ & 200 & 1000 & 2000 & 12 \\
\hline Vinho $=\mathbf{W}$ & 0,05 & 20 & 4,5 & 200 & 900 & 4000 & 10 \\
\hline Total & & & & & 1900 & 6000 & 22 \\
\hline & & & & & & & \\
\hline \multicolumn{8}{|l|}{ Restrições } \\
\hline \multicolumn{2}{|c|}{ Espaço disponível } & 15000 & & & & & \\
\hline \multicolumn{2}{|c|}{ Máximo de caixas de vasilham } & 800 & & & & & \\
\hline \multicolumn{2}{|c|}{ Total de horas disponível } & 60 & & & & & \\
\hline & & & & & & & \\
\hline \multicolumn{8}{|c|}{ A seguir : problema resolvido com os dados enunciados } \\
\hline $\begin{array}{l}\text { Tipo da } \\
\text { garrafa }\end{array}$ & $\begin{array}{c}\text { Horas de } \\
\text { trabalho para } \\
\text { cada caixa de } \\
\text { garrafas }\end{array}$ & $\begin{array}{c}\text { Espaço para } \\
\text { armazenar } \\
\text { cada caixa }\end{array}$ & $\begin{array}{c}\text { Contribuição } \\
\text { líquida por } \\
\text { caixa }\end{array}$ & $\begin{array}{l}\text { Número } \\
\text { de caixas }\end{array}$ & $\begin{array}{l}\text { Lucro total } \\
\text { de garrafas }\end{array}$ & $\begin{array}{c}\text { Espaço } \\
\text { necessário } \\
\text { total }\end{array}$ & $\begin{array}{c}\text { Total } \\
\text { horas } \\
\text { trabalhadas }\end{array}$ \\
\hline Suco = J & 0,06 & 10 & $\mathbf{5 , 0}$ & 643 & 1000 & 6.429 & 39 \\
\hline Vinho $=\mathbf{W}$ & 0,05 & 20 & 4,5 & 429 & 900 & 8.571 & 21 \\
\hline Total & & & & & $5.142,86$ & 15.000 & 60 \\
\hline
\end{tabular}


O $5^{\circ}$ problema, o Sr. Tanakara Kenfoi, com base no inventário existente em sua empresa, deseja produzir produtos acabados de modo a maximizar seu lucro.

O Sr. Tanakara Kenfoi gerencia uma fábrica que produz 3 produtos :

TV's, Aparelhos de som Stereo e speakers.Cada produto é montado de partes do estoque e há 5 tipos de componentes : chassis, tubos de imagem, alto falantes, caixa de força e partes eletrônicas. $O$ objetivo é produzir um conjunto de produtos que devem maximizar os lucros, dados o inventário de componentes existentes.

Para montar uma TV, são necessários : 1 chassis, 1 tubo de imagem, 2 alto-falantes, 1 caixa de força e 2 conjuntos de partes eletrônicas. Para montar um aparelho de som stered são necessários : 1 chassis, 2 alto-falantes, 1 caixa de força e 1 conjunto de partes eletrônicas Para montar um speaker, são necessários somente : 1 alto-flante e 1 conjunto de partes ele trônicas. Cada aparelho de TV é vendido com um GPM de \$75 cada, aparelhos de som com GPM de $\$ 50$ cada e speakerspor $\$ 35$ cada.

$O$ inventário existente é de $\mathbf{4 5 0}$ chassis, 250 tubos de imagem, 800 alto-falantes, caixas de força e 600 conjuntos de partes eletrônicas.

Formulação algébrica :

x1 = quantidade de TV's montadas;

x2 = quantidade de aparelhos de som;

x3 = quantidade de speakers

Objetivo : maximizar o lucro de : 75.x1 + 50.x2 + 35.x3

Restrições :

$1 . \times 1+1 . \times 2+0 . \times 3<=450($ chassis $)$

$1 . x 1+0 . x 2+0 . x 3<=250$ ( tubos de imagem)

$2 . x 1+2 . x 2+1 . x 3<=800$ (alto-falantes)

$1 . x 1+1 . x 2+0 . x 3<=450$ (caixa de força)

$2 . x 1+1 . x 2+1 . x 3<=600$ (partes eletrônicas)

\begin{tabular}{|c|c|c|c|c|c|}
\hline & & & TV's & Som & Speaker \\
\hline & \multicolumn{2}{|c|}{ Quantidades a serem montadas } & 100 & 100 & 100 \\
\hline Componentes & Inventário & Quant. Usada & & & \\
\hline chassis & 450 & 200 & 1 & 1 & $\mathbf{0}$ \\
\hline tubo de imagem & 250 & 100 & 1 & $\mathbf{0}$ & $\mathbf{0}$ \\
\hline alto-falante & 800 & 500 & 2 & 2 & 1 \\
\hline caixa de força & 450 & 200 & 1 & 1 & 0 \\
\hline \multirow[t]{6}{*}{ parte eletrônica } & 600 & 400 & 2 & 1 & 1 \\
\hline & & Lucro & & & \\
\hline & & por produto & 75 & 50 & 35 \\
\hline & Total & 16000 & & & \\
\hline & & & TV's & Som & Speaker \\
\hline & \multicolumn{2}{|c|}{ Quantidades a serem montadas } & 200 & 200 & 0 \\
\hline Componentes & Inventário & Quant. Usada & & & \\
\hline chassis & 450 & 400 & 1 & 1 & $\mathbf{0}$ \\
\hline tubo de imagem & 250 & 200 & 1 & $\mathbf{0}$ & $\mathbf{0}$ \\
\hline alto-falante & 800 & 800 & 2 & 2 & 1 \\
\hline caixa de força & 450 & 400 & 1 & 1 & 0 \\
\hline \multirow[t]{4}{*}{ parte eletrônica } & 600 & 600 & 2 & 1 & 1 \\
\hline & & Lucro & & & \\
\hline & & por produto & 75 & 50 & 35 \\
\hline & Total & 25000 & & & \\
\hline
\end{tabular}


O $6^{\circ}$ problema foi solicitado em um trabalho tipo “Atividade Estruturada” em uma Universidade em São Paulo, com a finalidade de maximizar o lucro da produção da fábrica.

\section{Problema de Pesquisa Operacional com Solver}

Uma fábrica produz marobus, marobos e marobis pelos processos alfa e beta a partir de insumos (recursos) : pequebes e pequebas.

$O$ processo alfa usa, por fornada, 2 unidades de pequebes e 5 de pequebas para produzir 20 marobus, 25 marobos e 30 marobis.

$O$ processo beta usa, por fornada, 4 unidades de pequebes e 5 de pequebas para produzir 23 marobus, 27 marobos, 35 marobis.

Os lucros são de 10 unidades monetárias por fornada do processo alfa e 15 unidades mone tárias do processo beta.

Dispõe-se de 150 pequebes e de 300 pequebas.

O sistema de fabricação exige que para cada fornada do processo beta sejam executadas, pelo menos, duas fornadas de alfa. É preciso produzir não mais de 3 marobis para cada marobus.

Obje tivando a maximização do lucro total, modele o problema, resolva-o através do "Solver do Excel"

Modelagem :

VD $\mathbf{A}=$ quantidade a ser produzida pelo processo alfa

B = quantidade a ser produzida pelo processo beta

Zmáx $=10 . A+15 . B$

S.a Disponibilidade de pequebes

Disponibilidade de pequebas

Disponibilidade de forno

Produção : marobis / marobus : 30 . A + 34 . B >= 0

(3)

$\tilde{\mathbf{n}} \mathbf{n} \quad: \mathbf{A}>=\mathbf{0} ; \mathbf{B}>=\mathbf{0}$

(1)
: $2 . A+4 . B<=150 \quad 3 / 1=(30 . A+35 . B) /(20 . A+23 . B)$

: $5 . A+5 . B<=30060 . A+69 . B<=30 . A+35 . B$

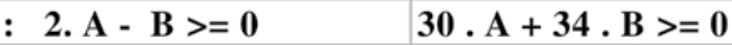

\begin{tabular}{|c|c|c|c|c|c|}
\hline & & & & & \\
\hline & & & & & \\
\hline & & & & & \\
\hline & & & & & \\
\hline$\underline{\text { Valor de Alfa }}$ & Valor de Beta & $\underline{\text { Lucro Z }}$ & & & \\
\hline 45 & 15 & 675 & & & \\
\hline & Alfa & Beta & Disponib. & Consumo & Sobras \\
\hline Pequebes & 2 & 4 & 150 & 150 & 0 \\
\hline Pequebas & 5 & 5 & 300 & 300 & 0 \\
\hline Demanda & 2 & -1 & 0 & 105 & -105 \\
\hline Demanda & 30 & 34 & 0 & 1860 & -1860 \\
\hline Lucro & 10 & 15 & 0 & 0 & 0 \\
\hline
\end{tabular}


O $7^{\circ}$ e último problema, deixamos para exercício dos leitores; trata-se do investidor chinês Sr. Pun Yat Chin, que possui um determinado valor a investir inicialmente e deseja formular um modelo que forneça um plano de investimento, informando quanto de dinheiro o investidor terá no ano seguinte.

O famoso investidor chinês Pun Yat Chin, lutador de Sai Korren Dô, tem 3 alternativas de investimento, disponíveis no próximo ano, elas não são mutuamente exclusivas; qualquer dinheiro recebido de qualquer alternativa poderá ser reinvestido, imediatamente, em qualquer uma das 3 alternativas. A alternativa A está disponível no princípio de cada um dos 4 trimestres seguintes, cada \$ investido em A no princípio de um trimestre devolve \$ 1,10 no final daquele trimestre.

A alternativa $B$ está disponível no princípio de cada um dos 2 semestres seguintes, cada \$ investido em B no princípio de um semestre devolve \$ 1,20 no final daquele semestre.

A alternativa $\mathbf{C}$ só está disponível no princípio do primeiro ano, cada $\$$ investido lhe devolve $\$ 1,40$ um ano mais tarde.

O capital inicial do Sr. Pun é de \$ 5.000,00. Deseja-se formular um modelo para fornecer o plano de investimento que maximize a quantidade de dinheiro que o investidor pode acumular no final do próximo ano.

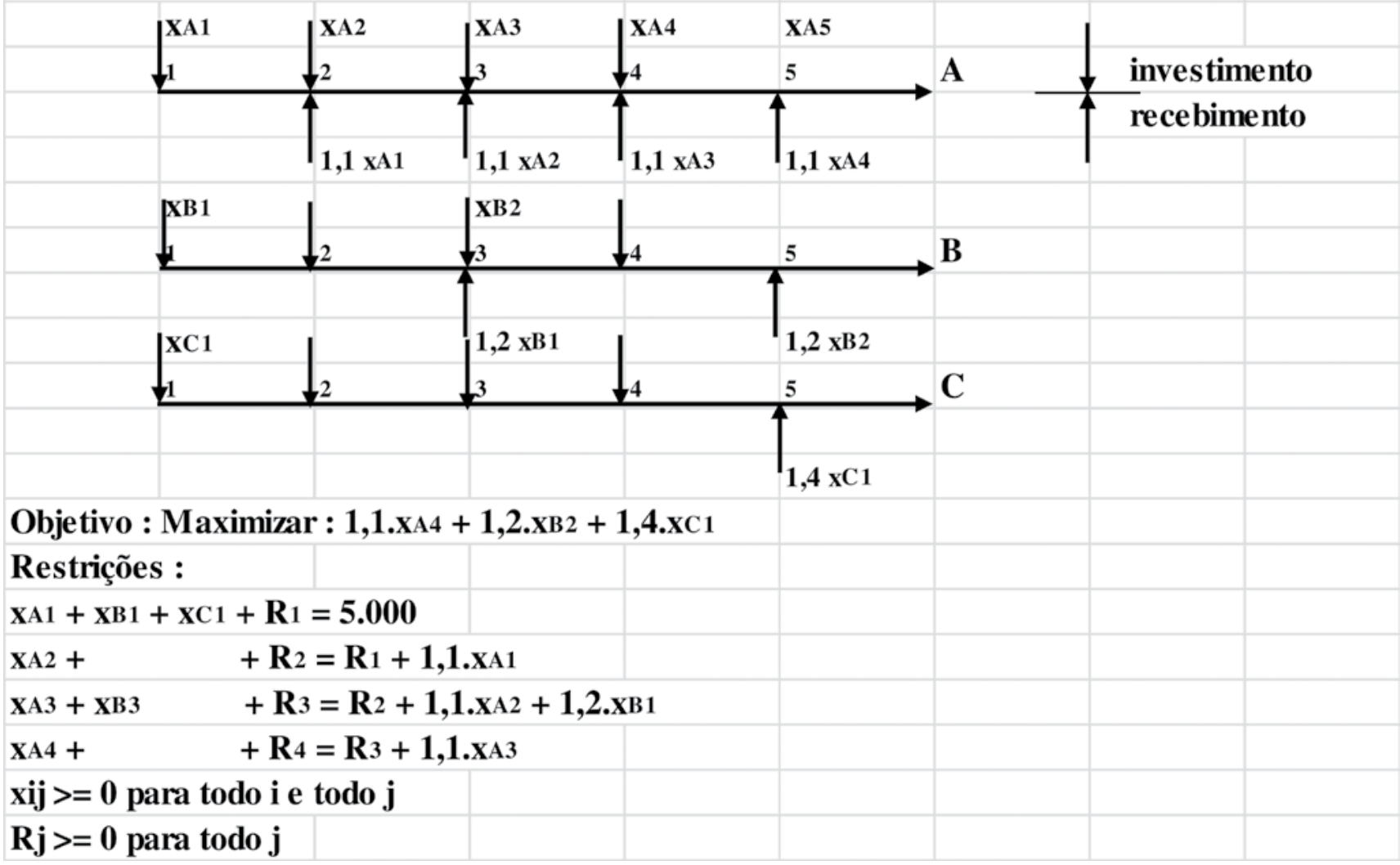


O SOLVER não termina pela solução do problema, ele apresenta ainda :

- um Relatório de Limites;

- um Relatório de Respostas e

- um Relatório de Sensibilidade

A seguir, cada destes relatórios para o $5^{\circ}$ problema :

\section{Microsoft Excel 8.0a Relatório de limites}

\begin{tabular}{lcc}
\hline Célula & $\begin{array}{c}\text { Destino } \\
\text { Nome }\end{array}$ & Valor \\
\hline$\$ C \$ 48$ & Total Por produto & 25000 \\
\hline
\end{tabular}

\begin{tabular}{llr}
\hline \multicolumn{1}{c}{$\begin{array}{c}\text { Ajus tável } \\
\text { Nome }\end{array}$} & Valor \\
Célula & \multicolumn{1}{c}{ 200 } \\
\hline \$D\$39 & Quantidades a serem montadas TV's & 200 \\
\hline \$E\$39 & Quantidades a serem montadas Som & Q \\
\hline \$F\$39 & Quantidades a serem montadas Speaker & 0 \\
\hline
\end{tabular}

\begin{tabular}{rr}
\hline $\begin{array}{c}\text { Inferior } \\
\text { Limite }\end{array}$ & $\begin{array}{c}\text { Destino } \\
\text { Resultado }\end{array}$ \\
\hline 0 & 10000 \\
\hline 0 & 15000 \\
\hline 0 & 25000 \\
\hline
\end{tabular}

\begin{tabular}{rr}
\hline $\begin{array}{c}\text { Superior } \\
\text { Limite }\end{array}$ & $\begin{array}{c}\text { Destino } \\
\text { Resultado }\end{array}$ \\
\hline 200 & 25000 \\
\hline 200 & 25000 \\
\hline 0 & 25000 \\
\hline
\end{tabular}

\section{Microsoft Excel 8.0a Relatório de resposta}

Célula de destino (Máx)

\begin{tabular}{lrrr}
\hline Célula & Nome & Valor original & \multicolumn{1}{c}{ Valor final } \\
\hline$\$ C \$ 48$ & Total Por produto & 16000 & 25000 \\
\hline
\end{tabular}

Células ajustáveis

\begin{tabular}{llrr}
\hline Célula & Nome & Valor original & \multicolumn{2}{c}{ Valor final } \\
\hline \$D\$39 & Quantidades a serem montadas TV's & 100 & 200 \\
\hline$\$ E \$ 39$ & Quantidades a serem montadas Som & 100 & 200 \\
\hline$\$ F \$ 39$ & Quantidades a serem montadas Speaker & 100 & 0 \\
\hline
\end{tabular}

Restrições

\begin{tabular}{|c|c|c|c|c|c|}
\hline Célula & Nome & Valor da célula & Fórmula & Status & Transigência \\
\hline$\$ C \$ 41$ & chassis Quant. Usada & 400 & $\mathrm{C} \$ 41<=\$ B \$ 41$ & Sem agrupar & 50 \\
\hline$\$ C \$ 42$ & tubo de imagem Quant. Usada & 200 & $\mathrm{C} \$ 42<=\$ B \$ 42$ & Sem agrupar & 50 \\
\hline$\$ C \$ 43$ & alto-falante Quant. Usada & 800 & $\mathrm{C} \$ 43<=\$ B \$ 43$ & Agrupar & 0 \\
\hline$\$ C \$ 44$ & caixa de força Quant. Usada & 400 & $\mathrm{C} \$ 44<=\$ B \$ 44$ & Sem agrupar & 50 \\
\hline$\$ C \$ 45$ & parte eletrônica Quant. Usada & 600 & $\mathrm{C} \$ 45<=\$ B \$ 45$ & Agrupar & 0 \\
\hline \$D\$39 & Quantidades a serem montadas TV's & 200 & $\mathrm{D} \$ 39>=0$ & Sem agrupar & 200 \\
\hline$\$ E \$ 39$ & Quantidades a serem montadas Som & 200 & $\mathrm{E} \$ 39>=0$ & Sem agrupar & 200 \\
\hline$\$ F \$ 39$ & Quantidades a serem montadas Speaker & & $F \$ 39>=0$ & Agrupar & 0 \\
\hline
\end{tabular}




\section{Micros oft Excel 8.0a Relatório de sensibilidade}

Células ajustáveis

\begin{tabular}{llrr}
\hline \multicolumn{1}{c}{ Nome } & $\begin{array}{c}\text { Final } \\
\text { Valor }\end{array}$ & $\begin{array}{l}\text { Reduzido } \\
\text { Gradiente }\end{array}$ \\
\hline \$D\$39 & Quantidades a serem montadas TV's & 200 & 0 \\
\hline \$E\$39 & Quantidades a serem montadas Som & 200 & 0 \\
\hline \$F\$39 & Quantidades a serem montadas Speaker & 0 & $-2,499975145$ \\
\hline
\end{tabular}

Restrições

\begin{tabular}{llcr}
\hline \multicolumn{1}{c}{ Nome } & \multicolumn{2}{c}{ Final } & \multicolumn{2}{c}{ Lagrange } \\
Célula & Valor Multiplicador \\
\hline \$C\$41 & chassis Quant. Usada & 400 & 0 \\
\hline \$C\$42 & tubo de imagem Quant. Usada & 200 & 0 \\
\hline \$C\$43 & alto-falante Quant. Usada & 800 & 12,5 \\
\hline \$C\$44 & caixa de força Quant. Usada & 400 & 0 \\
\hline \$C\$45 & parte eletrônica Quant. Usada & 600 & 25 \\
\hline
\end{tabular}

\section{Considerações finais}

Como podem verificar, esta ferramenta do Excel é extremamente útil aos profissionais de Engenharia, seja a modalidade que for; certamente haverá uma oportunidade de aplicá-la em casos reais da empresa em que estiverem trabalhando.

Dedico este resumo aos universitários da Estácio de Sá, procurando mostrar-lhes que um aplicativo como o Excel pode ajudar a resolver problemas na sua vida profissional.

\section{Referências bibliográficas}

Hiller, Frederick S; Lieberman,Gerald J. ; Introdução à Pesquisa Operacional, Stanford University, Editora Campus, 1974.

Ragsdale, Cliff T. ; Spreadsheet Modeling and Decision Analysis, Virginia Polytechnic and State University, USA, 1998.

Shimizu, Tomio ; Pesquisa Operacional em Engenharia, Economia e Administração - Modelos Básicos e Métodos Computacionais, Editora Guanabara Dois, 1984.

Hirschfeld, Henrique; Engenharia Econômica, Editora Atlas, 1986. Lapponi, Juan Carlos; Modelagem Financeira com Excel e VBA, Editora Campus, 2008

Ellenrieder, Alberto von; Pesquisa Operacional, ITA, Editora Almeida Neves, 1971.

Bronson, Richard; Pesquisa Operacional, Editora McGraw-Hill, 1985. 